\section{MS5-P17 The effects of electrostatic interactions and side-chain stabilization during chorismate mutase-catalyzed Claisen rearrangements}

Daniel Burschowsky ${ }^{1}$, David Balcells ${ }^{1}$, Einar Uggerud ${ }^{1}$, Peter Kast $^{2}$, Ute Krengel ${ }^{1}$

1. Department of Chemistry, University of Oslo

2. Laboratory of Organic Chemistry, ETH Zurich

email: danielbu@kjemi.uio.no

The chorismate mutase (CM) of Bacillus subtilis is a well-characterized model enzyme that catalyzes the pericyclic rearrangement of chorismate to prephenate. However, the relative contributions of transition-state stabilization and geometry to its catalytic efficiency $\left(10^{6}\right.$-fold) were debated during the last decade. An almost inactive variant of $\mathrm{CM}$, where the charged catalytic residue Arg90 was replaced by a neutral citrulline, was supposed to resolve the debate, but the disagreement still persisted. Specifically, it was unclear if the observed changes in reaction energies for the inactive variant stemmed from large-scale structural changes or electrostatic effects. With a series of high-resolution crystallographic snapshots along its reaction coordinate we showed that the enzyme variant is structurally identical to wild-type $\mathrm{CM}$ and that the electrostatic stabilizing effect is therefore almost entirely responsible for catalysis.

The detailed experimental structural information of the main reaction states also allowed us to examine the active sites of the CM wild type and variant with quantum chemical simulations, without introducing conformational artifacts. Instead of using molecular dynamics to relax our structures beforehand, we were able to use the amino acid structures that comprise the active site environment directly. We optimized the geometries of truncated active site models in the presence of substrate, transition state and product for a range of dielectric constants and at different levels of density functional theory, which enabled us to confirm the electrostatic nature of $\mathrm{CM}$ catalysis. In addition, by following the intrinsic reaction coordinate of this model system, we were able to describe mechanistic details for this enzyme. Most prominently, a $13^{\circ}$ rotation of the catalytic side chain serves as an induced fit for a selective electrostatic stabilization of the transition state, which plausibly explains the catalytic proficiency of this enzyme.

1. Burschowsky D, et al. (2014) Electrostatic transition state stabilization rather than reactant destabilization provides the chemical basis for efficient chorismate mutase catalysis. Proc Natl Acad Sci U S A 111(49):17516-17521.

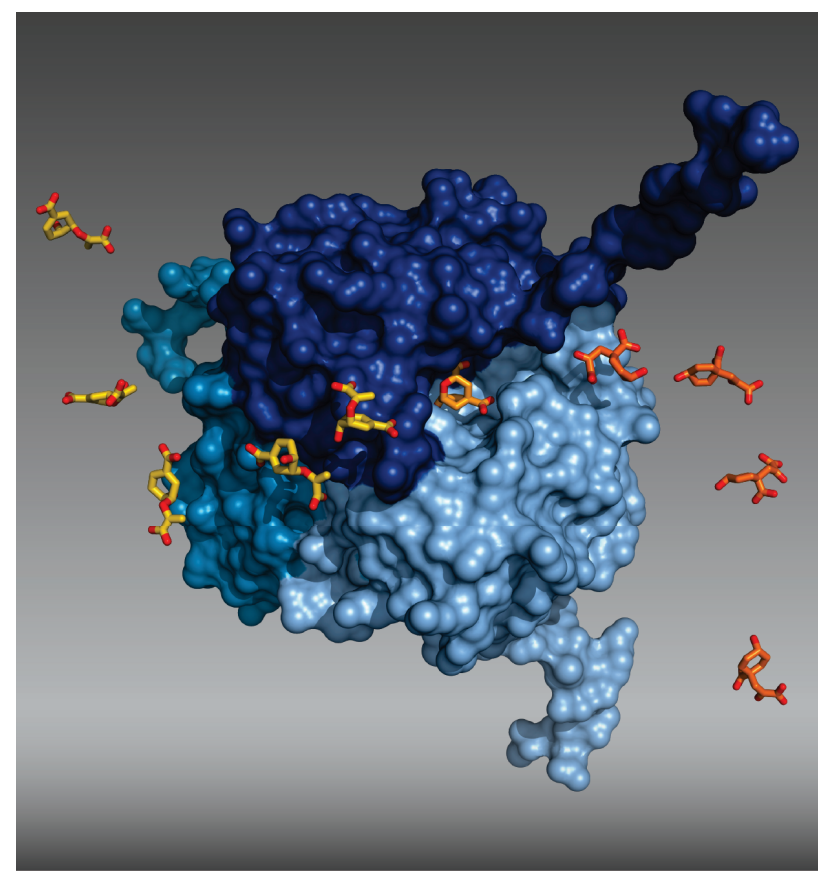

Figure 1. An artistic impression of the model enzyme chorismate mutase in action. Yellow colored chorismate molecules coming in from the left are transmuted via a bicyclic transition state (here depicted with a light orange transition state analog) to prephenate, shown in orange.

Keywords: enzyme mechanism, chorismate mutase, pericyclic reaction 